\title{
ARTICLE OPEN \\ Fiber-coupled EPR-state generation using a single temporally multiplexed squeezed light source
}

\author{
Mikkel V. Larsen (iD) ${ }^{1}$, Xueshi Guo (D) ${ }^{1}$, Casper R. Breum (D) ${ }^{1}$, Jonas S. Neergaard-Nielsen (iD) and Ulrik L. Andersen (iD
}

A prerequisite for universal quantum computation and other large-scale quantum information processors is the careful preparation of quantum states in massive numbers or of massive dimension. For continuous variable approaches to quantum information processing (QIP), squeezed states are the natural quantum resources, but most demonstrations have been based on a limited number of squeezed states due to the experimental complexity in up-scaling. The number of physical resources can however be significantly reduced by employing the technique of temporal multiplexing. Here, we demonstrate an application to continuous variable QIP of temporal multiplexing in fiber: Using just a single source of squeezed states in combination with active optical switching and a $200 \mathrm{~m}$ fiber delay line, we generate fiber-coupled Einstein-Podolsky-Rosen entangled quantum states. Our demonstration is a critical enabler for the construction of an in-fiber, all-purpose quantum information processor based on a single or few squeezed state quantum resources.

npj Quantum Information (2019)5:46; https://doi.org/10.1038/s41534-019-0170-y

\section{INTRODUCTION}

The realization of quantum computation (QC) with demonstrated quantum supremacy requires a scalable platform of quantum resources: ${ }^{1,2}$ Usually hundreds of logical qubits, or thousands of physical qubits, are needed to reach this longstanding goal. ${ }^{3}$ In one-way measurement-based quantum computation (MBQC), ${ }^{4,5}$ universal computation is performed with only single-qubit projective measurements of an entangled cluster state. ${ }^{6}$ Thereby, scalability is relaxed to the generation of a cluster state of suitable size. $^{7}$ Cluster states of multiple modes of light are readily accessible in continuous variable optical platforms, but most demonstrations have been limited by the amount of spatial resources. ${ }^{8-12}$ However, by time and frequency multiplexing with squeezed states of light, large cluster states can be deterministically generated as demonstrated with 60 frequency modes in refs ${ }^{13,14}$ and $10^{6}$ temporal modes in refs ${ }^{15,16}$ This allows for excellent scalability, thereby rendering the need for spatially distributed resources unnecessary.

MBQC based on temporally encoded cluster states ${ }^{17,18}$ from a single squeezed state resource ${ }^{19}$ requires optical switching and passive optical storage (such as an optical delay line) in different configurations as illustrated in Fig. 1. Multiple time-synchronized squeezed states can be generated in the network illustrated in Fig. 1a, allowing two-dimensional cluster state generation from a single squeezing source. ${ }^{17,18}$ Moreover, in MBQC, sequential measurements are performed on the cluster in which each measurement strategy is adaptively changed based on previous measurement outcomes. In some cases, switching between completely different measurement schemes, e.g., homodyne detection and a non-Gaussian measurement, is required ${ }^{18}$ (Fig. 1b). As an alternative to switching between Gaussian and non-Gaussian measurement schemes, one might fix the measurement setting to Gaussian homodyne detection and switch ancillary non-Gaussian states into selected modes of the cluster state $^{20}$ (Fig. 1C). Finally, it is possible to realize MBQC by applying optical switching in loop-based architectures ${ }^{21,22}$ as illustrated in Fig. 1d. No matter which of the strategies is chosen, switching and delay lines are key functionalities in managing temporal modes in optical MBQC.

In this article, we demonstrate optical fiber switching combined with an optical fiber delay in a continuous variable (CV) quantum setting in the telecom band. This enables us to generate an Einstein-Podolsky-Rosen (EPR) state ${ }^{23}$ between two fiber modes by time multiplexing of a single source of squeezed states of light. Our demonstration of optical switching and optical delay in a CV, fiber-integrated and low-loss setting is a critical step towards the realization of a scalable platform for CV quantum information processing and ultimately universal quantum computation.

\section{RESULTS}

The quadrature entangled EPR-state is an important resource in numerous quantum information and sensing protocols ranging from CV teleportation ${ }^{24}$ and cryptography ${ }^{25}$ to CV computing. ${ }^{26}$ The most wide-spread realization of quadrature entanglement is based on cavity-enhanced spontaneous parametric downconversion in an optical parametric oscillator (OPO). Correlations can be established between different polarization or frequency modes from a single non-degenerate $\mathrm{OPO},{ }^{27-34}$ or by combining the squeezed state outputs of two degenerate OPOs onto a balanced beam splitter. ${ }^{24,35-37}$ Here, we use the latter approach of combining two squeezed states on a beam splitter, but instead of using two OPOs, we exploit time multiplexing of a single source.

\section{Experimental set-up}

The experimental set-up is sketched in Fig. 2. We inject a single $\sim 7 \mathrm{~dB}$ squeezed beam into a fiber switch that alternately guides

${ }^{1}$ Center for Macroscopic Quantum States (bigQ), Department of Physics, Technical University of Denmark, Fysikvej, 2800 Kongens Lyngby, Denmark

Correspondence: Mikkel V. Larsen (mivila@fysik.dtu.dk) or Jonas S. Neergaard-Nielsen (jsne@fysik.dtu.dk) or Ulrik L. Andersen (ulrik.andersen@fysik.dtu.dk)

Received: 8 February 2019 Accepted: 7 June 2019

Published online: 08 July 2019 

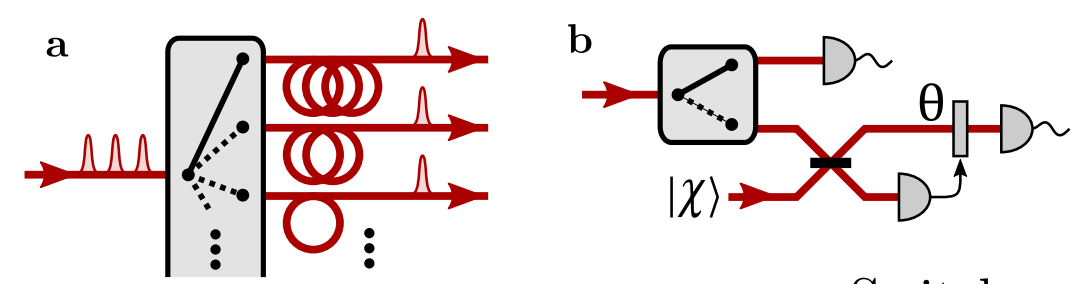

C

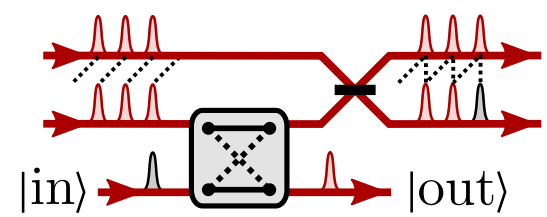

d

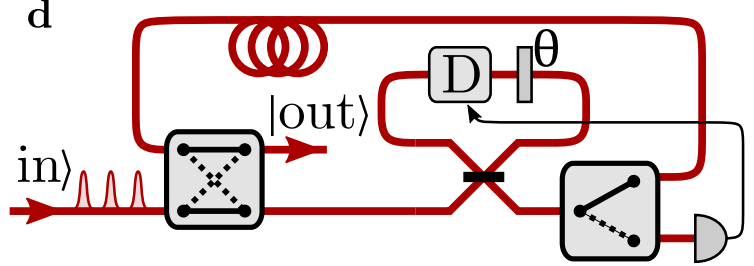

Switches

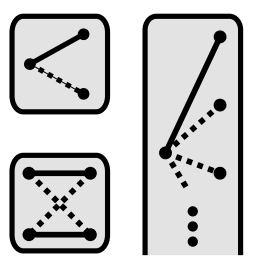

D Homodyne detector $\Lambda$ Temporal mode (O) Delay * Beam splitter (BS) BS transformation

Fig. 1 Quantum information processing architectures using optical switching and optical delay. a Switching and delay lines applied to a single squeezed state resource in order to generate multiple time-synchronized squeezed state. b Switching between homodyne detection and the more demanding cubic-phase gate-teleportation measurement with $|X\rangle$ being an ancillary cubic-phase-state. ${ }^{18} \mathbf{c}$ Example of switching temporal modes into or out of a cluster state. ${ }^{20} \mathbf{d}$ Loop-based architecture for fully temporally encoded MBQC utilizing switching and delay. ${ }^{21}$

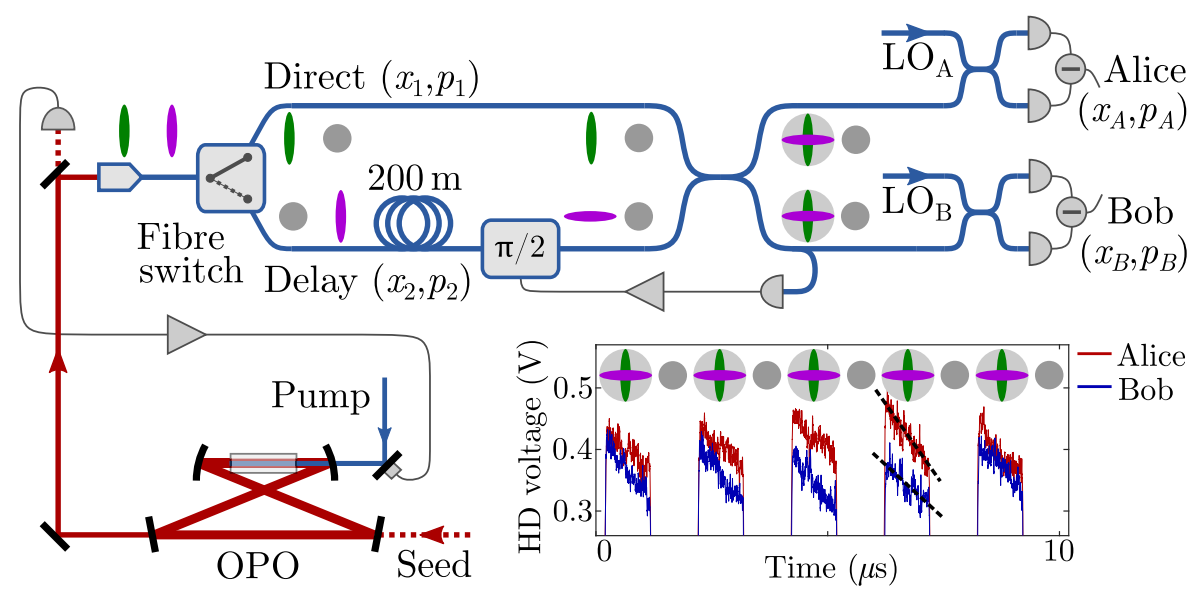

Fig. 2 Schematics of the experiment. Bright amplitude squeezed states of light are generated using type-0 parametric down-conversion in an optical resonator (OPO) at the wavelength of $1550 \mathrm{~nm}$, seeded with a coherent beam for phase locks. The squeezed states of light are coupled into a single mode fiber network (marked by blue lines) in which the generation of two-mode squeezing takes place: Using a fiber switch, two consecutive temporal modes (marked by green and purple) are guided in different directions. Subsequently, the two modes are synchronized by a fiber delay of $200 \mathrm{~m}$ in one of the modes. Finally, the two spatial modes interfere in a 50:50 fiber coupler, thereby forming a two-mode squeezed state in the output as the phase difference of the two input modes are locked to $\pi / 2$ (using active feedback to a fiber-stretching device described in Methods). The quadratures of the two-mode squeezed state are measured with two fiber-based homodyne detection stations, Alice and Bob. A typical measurement output in time-domain is shown in the inset together with illustrations of the corresponding states in phase space, alternating between two-mode squeezed states and vacuum states

the squeezed beam into two different fibers at a frequency of $500 \mathrm{kHz}$; thereby delaying one mode by $1 \mu \mathrm{s}$ with respect to the other. To compensate for the delay and thus synchronize the two modes in time, the mode ahead propagates through a $200 \mathrm{~m}$ fiber spool. The two modes interfere with a relative phase shift of $\pi / 2$ in a balanced fiber coupler, thereby forming a two-mode squeezed state.

For state characterization, we sample on an oscilloscope the quadratures of the fiber coupler outputs measured by two homodyne detection stations, Alice and Bob. Typical time traces of such measurements are shown in the inset of Fig. 2. A single dataset consists of 16,000 time traces triggered by the switching signal. Each time trace is affected by a frequency-dependent response of the detector giving rise to the negative slope seen in the inset of Fig. 2, and a noisy oscillatory response of the fiber switch. Besides this, there is a variation in the slope of each time trace due to spurious interferences-both effects occur from the coherent amplitude of the initial bright squeezed state together with limited detection, switching and feedback bandwidths. However, since these effects are systematic, repeatable and synchronized with the switching process, they can be tracked and compensated in the data processing - see Methods.

We have striven to reduce the loss of all components to maintain as much of the non-classicality as possible. We used an antireflection coated graded-index lens to couple the squeezed light into the fiber with an efficiency of $97 \%$ (by matching counterpropagating light in the OPO cavity), we spliced together all fiber components to minimize fiber-to-fiber coupling losses and by using the wavelength of $1550 \mathrm{~nm}$, fiber propagation loss was negligible: Even through the $200 \mathrm{~m}$ fiber delay (standard SMF-28e + fiber), the propagation loss is $\leq 1 \%$. The largest loss contribution is caused by the fiber switch (Nanona by Boston Applied Technologies Inc.), where light is coupled into a bulk electro-optic material and back into fiber leading to $17 \%$ loss. Including OPO escape efficiency, detection efficiency, and various tapping for phase locks, the total 


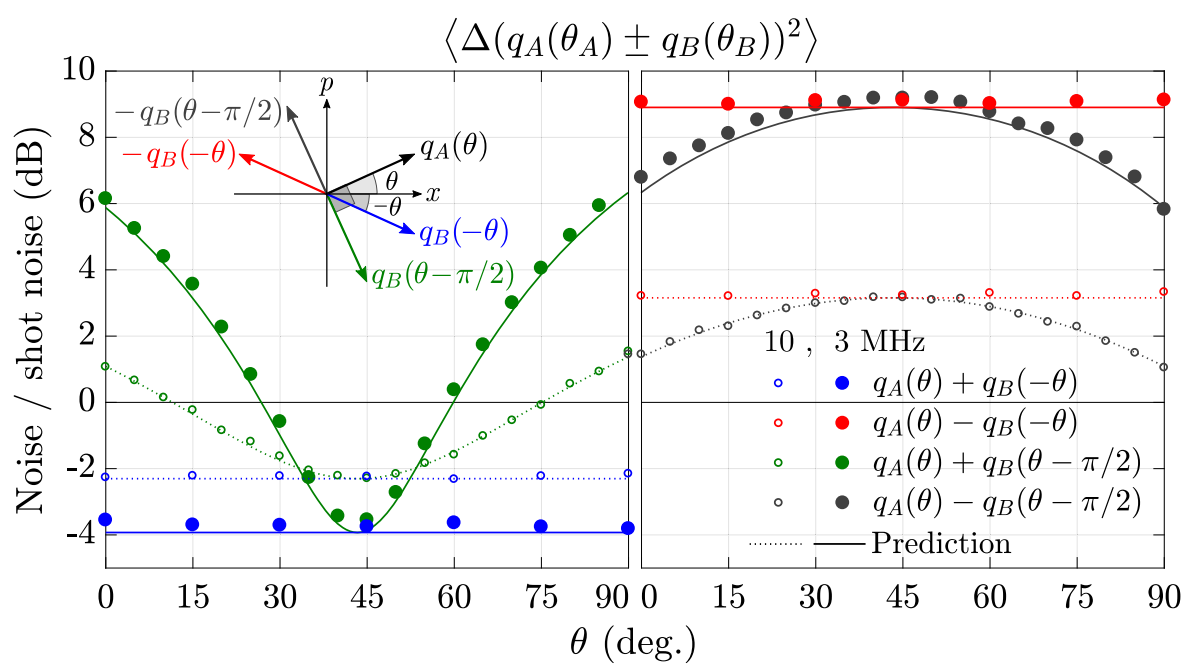

Fig. 3 Partial tomography of the generated two-mode squeezed state. We plot the noise variance (normalized to the shot noise variances) of the quadratures $q_{A}\left(\theta_{A}\right) \pm q_{B}\left(\theta_{B}\right)$ with $\theta_{A}$ restricted to $\theta_{A}=\theta$ and $\theta_{B}$ restricted to $\theta_{B}=-\theta$ (blue and red), and $\theta_{B}=\theta-\pi / 2$ (green and black). Each point corresponds to one dataset of 16,000 processed time traces as in Fig. 5 . To extract the 3 and $10 \mathrm{MHz}$ frequency modes, each time trace is digitally mixed with a 3 or $10 \mathrm{MHz}$ sine curve and integrated to one value. The noise is then the variance of these 16,000 values, added/ subtracted for Alice and Bob. With the time trace length of about $900 \mathrm{~ns}$, the frequency mode bandwidth is around $1 \mathrm{MHz}$. The solid and dashed line shows theoretical noise predicted from measured efficiency, OPO bandwidth, pump power, and fitted phase fluctuations in Fig. 4. The predictions include $1.7^{\circ}$ phase offset. The inset illustrates the measured quadratures in a phase-space diagram

transmission from the squeezed state source to the detected signal becomes $\eta \approx 68 \%$ (for more details see Methods).

\section{Experimental results}

To perform partial tomography of the generated two-mode squeezed states, we measure the quadratures $q_{A}(\theta) \pm q_{B}(-\theta)$ and $q_{A}(\theta) \pm q_{B}(\theta-\pi / 2)$ as a function of the local oscillator phase $\theta$. Here, $q_{i}(\theta)=x_{i} \cos \theta+p_{i} \sin \theta$ where $x_{i}$ is the amplitude and $p_{i}$ the phase quadrature at Alice $(i=A)$ and $\operatorname{Bob}(i=B)$. The resulting noise variances at the 3 and $10 \mathrm{MHz}$ side band frequencies are shown in Fig. 3 together with theoretical predictions. We observe a maximum shot noise suppression of $3.8 \mathrm{~dB}$. The very small discrepancy of the measurements at $3 \mathrm{MHz}$ results from technical noise of the seed beam as well as additional noise added in the delay line-both noise effects are discussed and analyzed below and in supplementary information section 3 .

The variances of $q_{A}(\theta) \pm q_{B}(-\theta)$,

$$
\begin{aligned}
& \left\langle\Delta\left(q_{A}(\theta)+q_{B}(-\theta)\right)^{2}\right\rangle=2\left(\left\langle\Delta x_{1}^{2}\right\rangle \cos ^{2} \theta+\left\langle\Delta x_{2}^{2}\right\rangle \sin ^{2} \theta\right), \\
& \left\langle\Delta\left(q_{A}(\theta)-q_{B}(-\theta)\right)^{2}\right\rangle=2\left(\left\langle\Delta p_{2}^{2}\right\rangle \cos ^{2} \theta+\left\langle\Delta p_{1}^{2}\right\rangle \sin ^{2} \theta\right),
\end{aligned}
$$

associated with the maximally squeezed and anti-squeezed quadratures, respectively, are seen to be constant with $\theta$, indicating symmetric two-mode squeezing. This is expected as the individual single mode squeezed states in the direct $\left(x_{1}, p_{1}\right)$ and delay $\left(x_{2}, p_{2}\right)$ line originate from the same squeezing source, that is $\left\langle\Delta x_{1}^{2}\right\rangle=\left\langle\Delta x_{2}^{2}\right\rangle$ and $\left\langle\Delta p_{1}^{2}\right\rangle=\left\langle\Delta p_{2}^{2}\right\rangle$. From the datasets at $\theta=$ $0^{\circ}$ and $90^{\circ}$, entanglement can be verified by the inseparability criterion, ${ }^{38}$ which reads

$$
\left\langle\Delta\left(x_{A}+x_{B}\right)^{2}\right\rangle+\left\langle\Delta\left(p_{A}-p_{B}\right)^{2}\right\rangle=1.72 V_{0}<4 V_{0} \text {, }
$$

at $3 \mathrm{MHz}$, and $2.42 V_{0}<4 V_{0}$ at $10 \mathrm{MHz}$. Here, $V_{0}$ is the variance of the vacuum state.

When measuring the variances of

$$
q_{A}(\theta) \pm q_{B}(\theta-\pi / 2)=x_{A} \cos \theta \pm x_{B} \sin \theta+p_{A} \sin \theta \mp p_{B} \cos \theta
$$

as a function of $\theta$, we trace out one specific projection that in particular realizes the squeezed and anti-squeezed quadratures.
Maximum squeezing and anti-squeezing are measured at $\theta=45^{\circ}$ where correlations are strongest, corresponding to the measurements of $q_{A}(\theta) \pm q_{B}(-\theta)$. At $\theta=0^{\circ}$ and $90^{\circ}$ we expect no correlations and measure the variances $\left(\left\langle\Delta x_{1}^{2}\right\rangle+\left\langle\Delta p_{1}^{2}\right\rangle+\left\langle\Delta x_{2}^{2}\right\rangle+\left\langle\Delta p_{2}^{2}\right\rangle\right) / 2$ corresponding to the added noise of thermal states at Alice and Bob when tracing out one mode.

From the partial tomography, we reconstruct the covariance matrix of the two-mode squeezed state at the $3 \mathrm{MHz}$ side band frequency: ${ }^{39}$

$$
\boldsymbol{v}=V_{0}\left(\begin{array}{cccc}
4.36 & - & -3.84 & 0.36 \\
- & 4.43 & 0.45 & 3.92 \\
-3.84 & 0.45 & 4.17 & - \\
0.36 & 3.92 & - & 4.26
\end{array}\right)
$$

Here, the entries with ' - ' were not measured as it would require a more elaborate measurement scheme, but they should in principle be zero due to the symmetry of the states. However, due to uncertainties in the phase control and non-perfect phasespace alignments, the values will in practice be slightly different from zero. This is also clear from the off-diagonal correlation terms $\left\langle x_{A} p_{B}\right\rangle$ and $\left\langle x_{B} p_{A}\right\rangle$, which in practice are non-zero as seen in the measured co-variance matrix but in theory should be zero for a perfectly aligned system (see supplementary information section 4). Finally, from the covariance matrix we determine the conditional variances between Alice and Bob's measurements from which we test the EPR-criterion: ${ }^{40}$

$$
\begin{aligned}
& \Delta_{\text {inf. }}^{2} x_{A \mid B} \cdot \Delta_{\text {inf. }}^{2} p_{A \mid B}=0.69 V_{0}^{2}<V_{0}^{2}, \\
& \Delta_{\text {inf. }}^{2} x_{B \mid A} \cdot \Delta_{\text {inf. }}^{2} p_{B \mid A}=0.64 V_{0}^{2}<V_{0}^{2},
\end{aligned}
$$

where $\Delta_{\text {inf. }}^{2} q_{i j}=\min _{g}\left\langle\Delta\left(q_{i}-g q_{j}\right)^{2}\right\rangle=\left\langle\Delta q_{i}^{2}\right\rangle-\left\langle q_{i} q_{j}\right\rangle^{2} /\left\langle\Delta q_{j}^{2}\right\rangle$ is the conditional uncertainty in predicting $q_{i}$ when measuring $q_{j}$. Since both conditional variance products are below $V_{0}^{2}$, the generated states are EPR entangled in both directions.

As seen from Eq. (1) for $\theta=0^{\circ}$ and $90^{\circ}$, the measured two-mode squeezing is equivalent to the squeezing of the single mode states in the direct and delayed paths, respectively. The spectra of such 


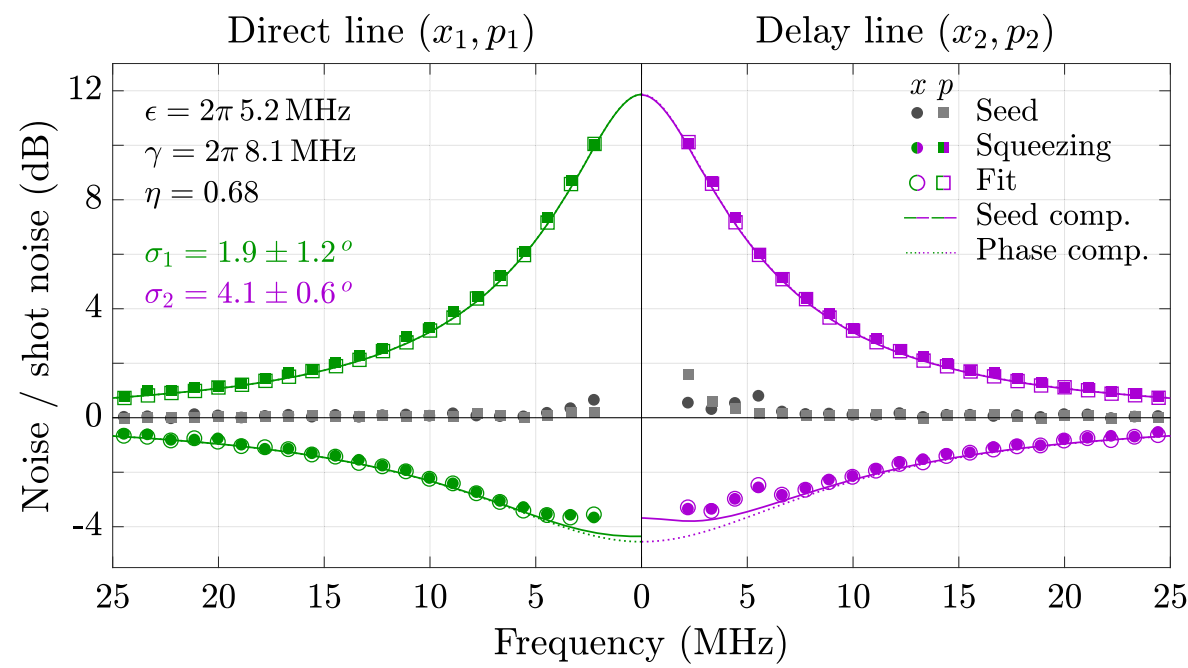

Fig. 4 Spectrum of squeezing. Noise spectrum of $x_{1}=\left(x_{A}+x_{B}\right) / \sqrt{2}, p_{1}=\left(p_{A}+p_{B}\right) / \sqrt{2}, x_{2}=\left(p_{A}-p_{B}\right) / \sqrt{2}$ and $p_{2}=\left(x_{A}-x_{B}\right) / \sqrt{2}$ relative to shot noise. Solid points correspond to the average of Fourier transformed time traces in the measured datasets $q_{A}(\theta) \pm q_{B}(-\theta)$ for $\theta=0^{\circ}$ and $90^{\circ}$. Here, colored points are with pumped OPO, while gray points are the seed noise when blocking the pump. Hollow points are the result of fitting a squeezing spectrum with phase fluctuations $\sigma_{1}$ and $\sigma_{2}$ in the direct and delay line, respectively. From the fit, the solid lines indicates the expected squeezing when compensating for seed noise, while the dashed lines indicates the expected squeezing in case of no phase fluctuation, and thus the best squeezing achievable with the given efficiency

measurements are shown in Fig. 4. The squeezing spectra are Lorentzian and resemble that of the OPO cavity. Furthermore, the anti-squeezing is seen to be symmetric, while the squeezing has degraded slightly in the delay line due to additional phase noise. To characterize this, we measure the seed spectrum by blocking the pump to the squeezing cavity. The low-frequency noise that can be observed in the direct line results from technical noise of the seed beam. Even more low-frequency noise is apparent in the squeezed state of the delay line. We believe it originates from phase noise generated by the $200 \mathrm{~m}$ fiber and amplitude noise from the fiber switch, which is most prominent at $5-6 \mathrm{MHz}$.

To infer the phase fluctuations, $\sigma_{i}$, associated with the direct $(i=1)$ and delay $(i=2)$ line, the squeezing spectra including a normal distributed phase with $\sigma_{i}$ standard deviation, approximated to $\left\langle\Delta x_{i}^{2}\right\rangle \cos ^{2}\left(\theta+\sigma_{i}\right)+\left\langle\Delta p_{i}^{2}\right\rangle \sin ^{2}\left(\theta+\sigma_{i}\right)$ for $\theta=0$ and $\pi / 2,{ }^{41}$ is fitted with $\sigma_{i}$ as the only fitting parameter. Here, following ${ }^{42}$ with additional seed noise coupled into the OPO and $V_{0}=1 / 2$,

$$
\left\langle\Delta q_{i}^{2}\right\rangle=\frac{1}{2} \mp \frac{2 \varepsilon \gamma \eta}{(\gamma \pm \varepsilon)^{2}+\omega^{2}}+\frac{K_{q}}{(\gamma \pm \varepsilon)^{2}+\omega^{2}}, q=x, p,
$$

where $\varepsilon$ is the pump rate, $\gamma$ is the total OPO decay rate, $\eta$ is the overall efficiency and $\omega$ is the angular frequency, while $K_{q}=$ $4 \gamma \gamma_{s} \eta_{i}\left(\left\langle\Delta q_{s}^{2}\right\rangle-1 / 2\right)$ with $\gamma_{s}$ being the decay rate due to the seed beam coupling mirror and $\left\langle\Delta q_{s}^{2}\right\rangle$ is the seed beam quadrature noise before injection into the OPO (for detailed derivation see supplementary information section 3.1). We find a decay rate of $\gamma /$ $2 \pi=8.1 \mathrm{MHz}$ by measuring the OPO intracavity losses $(0.55 \%)$, the cavity length $(320 \mathrm{~mm})$ and the transmissivity of the coupling mirror (10\%), and we estimate the pump rate to $\varepsilon / 2 \pi=5.2 \mathrm{MHz}$ for a pump power of $350 \mathrm{~mW}$ and a measured OPO threshold power of $833 \mathrm{~mW} . K_{q}$ is estimated as $K_{q}=\left(\gamma^{2}+\omega^{2}\right)\left(\left\langle\Delta q_{0}^{2}\right\rangle-1 / 2\right)$ where $\left\langle\Delta q_{0}^{2}\right\rangle$ is the quadrature noise measured with no pump ( $\varepsilon=0$, gray points in direct line of Fig. 4). Finally, to include excess noise of the delay line, the seed noise difference of the direct and delay line is added to the fit in the delay line. The fit is shown as hollow points in Fig. 4, and is seen to fit very well with the measured data. The resulting phase fluctuations obtained from the fit are $\sigma_{1}=1.9 \pm$ $1.2^{\circ}$ and $\sigma_{2}=4.1 \pm 0.6^{\circ}$ with uncertainties estimated as the $95 \%$ confidence interval. These values are included in the theoretical model used for Fig. 3.
From the theoretical model with fitted phase fluctuations, the solid lines in Fig. 4 indicate the expected squeezing spectra if the seed beam were shot noise limited and no additional noise existed in the delay line. In that case, we can expect more than $4 \mathrm{~dB}$ two-mode squeezing. The phase fluctuation in the delay line, $\sigma_{2}=4.1 \pm 0.6^{\circ}$, is more than double that in the direct line, $\sigma_{1}=$ $1.9 \pm 1.2^{\circ}$. This is mainly due to limited phase control bandwidth of the fiber delay and low signal-to-noise ratio of the feedback signal. Finally, the dotted line in Fig. 4 shows the squeezing spectrum we would expect if we had perfect phase control, and thus the optimum squeezing we may measure with the given efficiency.

\section{DISCUSSION}

The fast switching frequency of $500 \mathrm{kHz}$ demonstrated here is suitable for encoding temporal modes of megahertz bandwidth and is thus applicable in the optical schemes in Fig. 1. Similarly, the low loss of the $200 \mathrm{~m}$ fiber allows for an efficient delay of almost $1 \mu \mathrm{s}$, compatible with the temporal modes defined by the switching. However, the $17 \%$ loss of the particular switch used here, as well as the phase fluctuations of $4^{\circ}$ standard deviation in the fiber delay, leads to decoherence and results in some limitations when used in quantum settings: For cluster state generation from a temporal multiplexed source, as in Fig. 1a, or when switching modes in and out of a cluster state, as in Fig. 1c, the switching loss and phase fluctuation leads to limited entanglement even when large amount of initial squeezing is available. Yet, it does not accumulate through the cluster state as the loss and phase fluctuation on each mode is local, and so it does not limit the cluster state size. It will be more detrimental in loop-based architectures, as in Fig. 1d, where a temporal mode passes through the same switch and delay line multiple times, and so the switch efficiency and delay phase fluctuations limit the number of passes possible and thereby the computation depth.

High-efficient fast switching is demonstrated in free-space, ${ }^{43}$ while one can imagine more compact fiber-coupled switching based on Mach-Zehnder interferometry. However, in either case care must be taken not to compromise the high switching frequency, as this leads to longer delay lines necessary and thereby larger phase fluctuations. In work towards temporal 


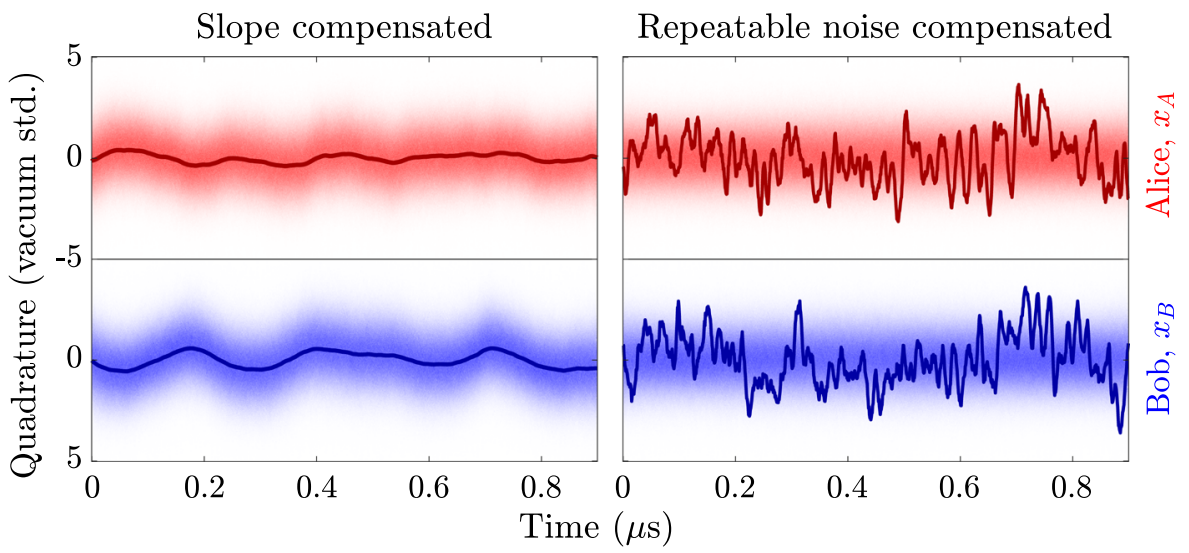

Fig. 5 Temporal filtering by data processing. (left) Temporal histogram of a dataset with 16,000 time traces associated with amplitude quadratures of a two-mode squeezed state at Alice (red) and Bob (blue) compensated for slope variations and decaying detector response. The solid lines show the dataset average time trace indicating the remaining repeating oscillations from the switching process. (right) Temporal histogram of the dataset in (left) compensated for any systematic and repeatable noise responses from the switching process. Here, the solid lines indicate a single pair of synchronized time traces (at Alice and Bob) in which quadrature anti-correlations are visible (note the inverted axis on Bob)

encoded optical quantum information processing, faster switching is preferable as it minimizes the required delay lengths and increases the computational speed. Thus, the ideal switch, besides being efficient, is as fast as the detection or squeezing source bandwidth.

In conclusion, using a single squeezing source with optical switching and delay, we have successfully generated in-fiber EPRstates with nearly $4 \mathrm{~dB}$ of two-mode squeezing, characterized by fiber-coupled homodyne detection. Our set-up has great scalability potentials: Adding an additional delay line, it is possible to extend the set-up to generate one-dimensional cluster states, ${ }^{15,16}$ and by adding a multi-port switch and more delay lines, twodimensional cluster states ${ }^{17,18}$ can be generated from a single squeezing source. Moreover, by inserting the switch inside a loop, as in Fig. 1d, combined with dynamical control, various entangled states can be generated and in principle universal quantum computation can be realized. Since all switches and delay lines are fiber components, the set-up remains very small and flexible despite the increasing complexity in generating more complex states. Moreover, since fiber propagation losses are extremely low at the operating wavelength of $1550 \mathrm{~nm}$, decoherence is not a big issue despite the increasing number of fiber delays. The largest decoherence source in the current set-up is the optical switch, which introduces a loss of $17 \%$. However, with future developments of the optical switch, we expect that the in-fiber temporal multiplexing technique demonstrated here will play a significant role in reducing the resources in future large-scale photonic circuits for continuous variable quantum information processing, including quantum computing, ${ }^{7}$ quantum teleportation, ${ }^{44}$ distributed sensing, ${ }^{45}$ and multi-partite quantum key distribution.

\section{METHODS}

Squeezing source

The experimental set-up is outlined in Fig. 2. As squeezing source, we use an optical parametric oscillator (OPO) based on a periodically poled potassium titanyl phosphate (PPKTP) crystal in a bowtie shaped cavity, locked by a counter-propagating coherent beam. A pump beam at a wavelength of $775 \mathrm{~nm}$ is used to drive the parametric process and thus produce squeezed light at $1550 \mathrm{~nm}$ via type-0 phase matching. The OPO has a bandwidth of $\gamma / \pi=16 \mathrm{MHz}$. Stable phase locking at different stages of the experiment is facilitated by an excitation of the squeezed state, realized by injecting a bright seed beam into the OPO. To lock the phase of the input pump beam to the deamplification point of the parametric process, thereby producing amplitude squeezed states, we tap off and detect $1 \%$ of the excited squeezed beam for feedback to a piezo-mounted mirror in the pump beam. This, as well as all other feedback controls in the experiment, is realized by the open-source software package PyRPL ${ }^{46}$ running on Red Pitaya boards that integrate an FPGA system-on-chip with fast analog-to-digital and digital-to-analog converters.

In-fiber phase control

For locking the $\pi / 2$ relative phase difference when interfering the two beams of bright squeezed states in a balanced fiber coupler for EPR-state generation, $1 \%$ is tapped off one of the fiber coupler output arms, and fed back to a homemade fiber stretcher in the delay line based on ref. ${ }^{47}$ Here, using a piezoelectric actuator, a phase shift is induced by stretching the fiber. For more details, see the supplementary information section 2 . The optical transmission efficiency is near unity, as it simply depends on the fiber, which has negligible loss at $1550 \mathrm{~nm}$ wavelength. This allows highefficient in-fiber phase control, and the same design is used for phase control of the local oscillators in the homodyne detection.

\section{Fiber-coupled homodyne detection}

To detect quadratures of the in-fiber generated EPR-state, we developed a fiber-coupled homodyne detector (HD) where signal and local oscillator (LO) is interfered in a balanced fiber coupler before detection. For schematics and details, see supplementary information section 2 . This has the benefit of being mobile, and the visibility between signal and LO is easily optimized to near unity due to the single mode nature of the fiber used.

The fiber coupler is not exactly symmetric, but has a coupling ratio of $\sim 48: 52$. To compensate for this, the HD is balanced by attenuation in the fiber coupler output arm of stronger LO by inducing bending losses. With an asymmetry of $4 \%$ in the fiber coupler, after balancing this leads to $4 \%$ loss.

Finally, to couple and focus light from the fiber onto the HD photo diodes of $100 \mu \mathrm{m}$ diameter (Laser Components Nordic AB), anti-reflective coated graded-index (GRIN) lens are used in front of the diode, leading to a free-space waist diameter of $13 \mu \mathrm{m}$ at $5 \mathrm{~mm}$ from the GRIN lens facet. The quantum efficiency is measured to be $97 \%$, and so together with $4 \%$ loss from balancing and $99 \%$ visibility, the total HD efficiency achieved is $91 \%$.

\section{Overall efficiency}

With the OPO escape efficiency of $95 \%$, and $1 \%$ tapping for gain lock, the efficiency in free-space before fiber coupling is $94 \%$. In fiber, including $97 \%$ fiber coupling efficiency, $17 \%$ loss in the fiber switch and 1\% tapping for phase control, the efficiency is $80 \%$. Finally, with $91 \%$ detection efficiency, 
the overall efficiency becomes

$$
\eta=0.94 \cdot 0.80 \cdot 0.91=68 \% \text {. }
$$

\section{Temporal data processing}

To recover two-mode squeezing from the acquired time traces affected by a frequency-dependent detector response (leading to a negative slope), spurious interference (leading to slope variations) and an oscillating response from the switch, we use the statistic of 16,000 time traces in a dataset synchronized with the switching process. To compensate for the negative and varying slope of each time trace, linear regression lines (as the dashed lines in the inset of Fig. 2) are subtracted from each individual trace of the dataset. The result is shown in Fig. 5 (left). Here, the repeatable oscillating noise is visible, and compensated for by subtracting the average time trace of the dataset from every single time trace. The final processed dataset is seen in Fig. 5(right) with a constant temporal histogram and a single time trace at Alice and Bob showing anti-correlations as in ref. ${ }^{48}$ For detailed discussion on the data processing, see supplementary information section 2.1 .

\section{DATA AVAILABILITY}

Experimental data and analysis code is available on request.

\section{ACKNOWLEDGEMENTS}

The work was supported by the Danish National Research Foundation through the Center for Macroscopic Quantum States (bigQ, DNRF142), and the VILLUM FOUNDATION Young Investigator Program.

\section{AUTHOR CONTRIBUTIONS}

U.L.A., J.S.N., and M.V.L. conceived the experiment. J.S.N., X.G., and C.R.B. designed and built the squeezing source. M.V.L. performed the experiment and analyzed the data. All authors contributed to the manuscript.

\section{ADDITIONAL INFORMATION}

Supplementary information accompanies the paper on the npj Quantum Information website (https://doi.org/10.1038/s41534-019-0170-y).

Competing interests: The authors declare no competing interests.

Publisher's note: Springer Nature remains neutral with regard to jurisdictional claims in published maps and institutional affiliations.

\section{REFERENCES}

1. Nielsen, M. A. \& Chuang, I. L. Quantum Computation and Quantum Information. (Cambridge University Press, England, 2000).

2. Bennett, C. H. \& DiVincenzo, D. P. Quantum information and computation. Nature 404, 247 (2000).

3. Dalzell, A. M., Harrow, A. W., Koh, D. E. \& La Placa, R. L. How many qubits are needed for quantum computational supremacy? arXiv:quant-ph/1805.05224v2 (2018).

4. Gottesman, D. \& Chuang, I. L. Quantum teleportation as a universal computational primitive. Nature 402, 390 (1999).

5. Raussendorf, R. \& Briegel, H. J. A one-way quantum computer. Phys. Rev. Lett. 86, 5188 (2001).

6. Raussendorf, R., Browne, D. E. \& Briegel, H. J. Measurement-based quantum computation with cluster states. Phys. Rev. A 68, 022312 (2003).

7. Gu, M., Weedbrook, C., Menicucci, N. C., Ralph, T. C. \& van Loock, P. Quantum computing with continuous-variable clusters. Phys. Rev. A 79, 062318 (2009).

8. Glöckl, O. et al. Experiment towards continuous-variable entanglement swapping: highly correlated four-partite quantum state. Phys. Rev. A 68, 012319 (2003).

9. Su, X. et al. Experimental preparation of quadripartite cluster and GreenbergerHorne-Zeilinger entangled states for continuous variables. Phys. Rev. Lett. 98, 070502 (2007).

10. Dong, R. et al. An efficient source of continuous variable polarization entanglement. New J. Phys. 9, 410 (2007).
11. Yukawa, M., Ukai, R., van Loock, P. \& Furusawa, A. Experimental generation of four-mode continuous-variable cluster states. Phys. Rev. A 78, 012301 (2008).

12. Aoki, T. et al. Quantum error correction beyond qubits. Nat. Phys. 5, 541 (2009).

13. Roslund, J., Medeiros de Araújo, R., Jiang, S., Fabre, C. \& Treps, N. Wavelengthmultiplexed quantum networks with ultrafast frequency combs. Nat. Photonics $\mathbf{8}$, 109 (2014).

14. Chen, M., Menicucci, N. C. \& Pfister, O. Experimental realization of multipartite entanglement of 60 modes of a quantum optical frequency comb. Phys. Rev. Lett. 112, 120505 (2014).

15. Yoshikawa, J. et al. Invited article: generation of one-million-mode continuousvariable cluster state by unlimited time-domain multiplexing. APL Photonics 1, 060801 (2016).

16. Yokoyama, S. et al. Ultra-large-scale continuous-variable cluster states multiplexed in the time domain. Nat. Photonics 7, 982 (2013).

17. Menicucci, N. C. Temporal-mode continuous-variable cluster states using linear optics. Phys. Rev. A 83, 062314 (2011).

18. Alexander, R. N., Yokoyama, S., Furusawa, A. \& Menicucci, N. C. Universal quantum computation with temporal-mode bilayer square lattices. Phys. Rev. A 97, 032302 (2018).

19. Andersen, U. L., Gehring, T., Marquardt, C. \& Leuchs, G. 30 years of squeezed light generation. Phys. Scr. 91, 053001 (2016).

20. Alexander, R. N., Gabay, N. C., Rohde, P. P. \& Menicucci, N. C. Measurement-based linear optics. Phys. Rev. Lett. 118, 110503 (2017).

21. Takeda, S. \& Furusawa, A. Universal quantum computing with measurementinduced continuous-variable gate sequence in a loop-based architecture. Phys. Rev. Lett. 119, 120504 (2017).

22. Motes, K. R., Gilchrist, A., Dowling, J. P. \& Rohde, P. P. Scalable boson sampling with time-bin encoding using a loop-based architecture. Phys. Rev. Lett. 113, 120501 (2014).

23. Reid, M. D. et al. Colloquium: The Einstein-Podolsky-Rosen paradox: From concepts to applications. Rev. Mod. Phys. 81, 1727 (2009).

24. Furusawa, A. et al. Unconditional quantum teleportation. Science 282, 706 (1998).

25. Madsen, L. S., Usenko, V. C., Lassen, M., Filip, R. \& Andersen, U. L. Continuous variable quantum key distribution with modulated entangled states. Nat. Commun. 3, 1083 (2012).

26. Menicucci, N. C. et al. Universal quantum computation with continuous-variable cluster states. Phys. Rev. Lett. 97, 110501 (2006).

27. Ou, Z. Y., Pereira, S. F., Kimble, H. J. \& Peng, K. C. Realization of the EinsteinPodolsky-Rosen paradox for continuous variable. Phys. Rev. Lett. 68, 3663 (1992).

28. Bardroff, P. J. \& Stenholm, S. Two-mode laser with excess noise. Phys. Rev. A 62, 023814 (2000).

29. Schori, C., Sørensen, J. L. \& Polzik, E. S. Narrow-band frequency tunable light source of continuous quadrature entanglement. Phys. Rev. A 66, 033802 (2002).

30. Bowen, W. P., Treps, N., Schnabel, R. \& Lam, P. K. Experimental demonstration of continuous variable polarization entanglement. Phys. Rev. Lett. 89, 253601 (2002).

31. Hayasaka, K., Zhang, Y. \& Kasai, K. Generation of twin beams from an optical parametric oscillator pumped by a frequency-doubled diode laser. Opt. Lett. 29, 1665 (2004).

32. Laurat, J., Coudreau, T., Keller, G., Treps, N. \& Fabre, C. Effects of mode coupling on the generation of quadrature Einstein-Podolsky-Rosen entanglement in a type-II optical parametric oscillator below threshold. Phys. Rev. A 71, 022313 (2005).

33. Wenger, J., Ourjoumtsev, A., Tualle-Brouri, R. \& Grangier, P. Time-resolved homodyne characterization of individual quadrature-entangled pulses. Eur. Phys. J. D. 32, 391 (2005).

34. Villar, A. S., Martinelli, M., Fabre, C. \& Nussenzveig, P. Direct production of tripartite pump-signal-idler entanglement in the above-threshold optical parametric oscillator. Phys. Rev. Lett. 97, 140504 (2006).

35. Silberhorn, C. et al. Generation of continuous variable Einstein-Podolsky-Rosen entanglement via the Kerr nonlinearity in an optical fiber. Phys. Rev. Lett. 86, 4267 (2001).

36. Bowen, W. P., Schnabel, R., Lam, P. K. \& Ralph, T. C. Experimental investigation of criteria for continuous variable entanglement. Phys. Rev. Lett. 90, 043601 (2003).

37. Takei, N., Yonezawa, H., Aoki, T. \& Furusawa, A. High-fidelity teleportation beyond the no-cloning limit and entanglement swapping for continuous variables. Phys. Rev. Lett. 94, 220502 (2005).

38. Duan, L., Giedke, G., Cirac, J. I. \& Zoller, P. Inseparability criterion for continuous variable systems. Phys. Rev. Lett. 84, 2722 (2000).

39. Weedbrook, C. et al. Gaussian quantum information. Rev. Mod. Phys. 84, 621 (2012).

40. Reid, M. D. Demonstration of the Einstein-Podolsky-Rosen paradox using nondegenerate parametric amplification. Phys. Rev. A 40, 913 (1989).

41. Aoki, T., Takahashi, G. \& Furusawa, A. Squeezing at $946 \mathrm{~nm}$ with periodically poled KTiOPO4. Opt. Express 14, 6930 (2006). 
42. Collett, M. J. \& Gardiner, C. W. Squeezing of intracavity and traveling-wave light fields produced in parametric amplification. Phys. Rev. A 30, 1386 (1984).

43. Kaneda, F. \& Kwiat, P. G. High-efficiency single-photon generation via large-scale active time multiplexing. arXiv:quant-ph/1803.04803v1 (2018).

44. Pirandola, S., Eisert, J., Weedbrook, C., Furusawa, A. \& Braunstein, S. Advances in quantum teleportation. Nat. Photonics 9, 641 (2015).

45. Guo, X. et al. Distributed quantum sensing in a continuous variable entangled network. arXiv:quant-ph/1905.09408 (2019).

46. Neuhaus, L. et al. PyRPL (Python Red Pitaya Lockbox)—An open-source software package for FPGA-controlled quantum optics experiments. CLEO/Europe-EQEC IEEE http://www.pyrpl.org (2017).

47. Mei, H., Li, B., Huang, H. \& Rao, R. Piezoelectric optical fiber stretcher for application in an atmospheric optical turbulence sensor. Appl. Opt. 46, 4371 (2007).

48. Takei, N., Lee, N., Moriyama, D., Neergaard-Nielsen, J. S. \& Furusawa, A. Timegated Einstein-Podolsky-Rosen correlation. Phys. Rev. A 74, 060101 (2006).
Open Access This article is licensed under a Creative Commons Attribution 4.0 International License, which permits use, sharing, adaptation, distribution and reproduction in any medium or format, as long as you give appropriate credit to the original author(s) and the source, provide a link to the Creative Commons license, and indicate if changes were made. The images or other third party material in this article are included in the article's Creative Commons license, unless indicated otherwise in a credit line to the material. If material is not included in the article's Creative Commons license and your intended use is not permitted by statutory regulation or exceeds the permitted use, you will need to obtain permission directly from the copyright holder. To view a copy of this license, visit http://creativecommons. org/licenses/by/4.0/.

(c) The Author(s) 2019 